\title{
Celiac disease and migraine: is there a common backstage?
}

\author{
Raffaella Mormile
}

Accepted: 12 June 2014 / Published online: 26 June 2014

(C) Springer-Verlag Berlin Heidelberg 2014

\section{Dear Editor:}

Celiac disease (CD) is a chronic systemic immune-mediated condition triggered by dietary gluten in genetically susceptible individuals. Mucosal villous atrophy and crypt hyperplasia of the proximal small intestine represent the histopathological hallmark lesions of CD. Toxic gluten peptides have been found to elicit an important immune response in the celiac intestine after regiospecific deamidation by an endogenous extracellular enzyme, transglutaminase 2 (TG2). Among the proinflammatory cytokines involved in the enhanced expression of TG2, interferon-gamma (IFN- $\gamma$ ) has been demonstrated to be the most potent inducer of TG2 expression, followed by tumor necrosis factor alpha (TNF- $\alpha$ ). These two cytokines have been shown to upregulate TG2 mRNA expression synergistically by different signal transduction pathways. IFN- $\gamma$ mediated activation of TG2 seems principally to require phosphatidylinositol 3-kinase (PI3K) activity. On the other hand, it has been proved that signaling pathways induced by TNF- $\alpha$ include activation of p38 MAPK (mitogen-activated protein kinase). Although CD patients may suffer primarily from gastrointestinal symptoms, CD may be related to lots of extraintestinal disorders such as migraine. There has been evidence provided that there is a high frequency of migraines in patients with $C D$ and vice versa, with a beneficial effect of a gluten-free diet. Until today, it is unclear whether these conditions occur as consequence of a common underlying cause. Migraine is a neurovascular disease characterized by dysfunction of the cerebral nerves and blood vessels. The neuropeptide calcitonin gene-related peptide (CGRP) has been significantly implicated in the pathophysiology of migraine with an integral role. It has been suggested that the increased CGRP synthesis and release associated with migraine may be connected with the activation of MAPK pathways, which, in turn, can be modulated by endogenous inflammatory mediators such as TNF- $\alpha$. Concordantly, it has been verified that migraine patients tend to have increased TNF- $\alpha$ levels, compared with the control group. In addition, elevation of cerebrospinal fluid TNF- $\alpha$ levels has been reported in new daily persistent headache and treatment refractory chronic migraine. Each PI3K isoform has also been postulated to have a unique role in development of nociception and tissue inflammation. It has been proposed that PI3Ks display specificity with regard to neuronal type as well as to specific tissues. Furthermore, PI3K has been described as a key determinant in the establishment of central sensitization, the spinal cord phenomenon associated with persistent afferent inputs and contributing to chronic pain states. Taken together into account, we speculate that the co-occurrence of $\mathrm{CD}$ with migraine may be a true association. We advance the hypothesis that IFN- $\gamma$ and TNF- $\alpha$ cooperate to promote the development and progression of migraine in $\mathrm{CD}$ patients. We suppose that TNF- $\alpha$ determines susceptibility to migraine by increasing the amount of CGRP through activation of MAPK pathways. In turn, IFN- $\gamma$ causes migraine via activating PI3K signaling. Thus, patients suffering from migraine should be screened for $\mathrm{CD}$, assessing the effects of a gluten-free diet. Dual targeting of MAPK/PI3K pathways may be an effective strategy against $\mathrm{CD}$. Research studies are needed to verify whether synergistic inhibition of these two pathways may provide new insights into underlying molecular mechanisms and opportunities for therapeutic intervention in CD. 